\title{
Ocio, tiempo libre y voluntariado en personas mayores
}

\author{
Inmaculada Montero García* \\ Matías Bedmar Moreno**
}

\begin{abstract}
Resumen: Numerosos cambios se suceden a nivel mundial. El ocio, como elemento social y cultural, no permanece ajeno a tal evolución y, a su vez, contribuye desde el punto de vista personal, social y económico, a la configuración de determinados hábitos, estilos de vida, formas diferentes de entender el descanso y desarrollo recreativo de nuevas actividades. En este artículo se plantea el uso del ocio, tiempo libre y voluntariado, desde el colectivo de las personas mayores, destacando la importancia de su participación a través de distintas tareas. Esto supone una importante inversión de tiempo, conocimientos y apoyo afectivo, que no siempre es reconocido ni valorado por el resto de la sociedad. Concluimos destacando cómo la acción educativa a través de programas de ocio ha modificado su concepción y se define a través de parámetros que hacen referencia a la autonomía, desarrollo, relación, participación y creatividad, también en las personas de más edad.

Palabras clave: Ocio, tiempo libre, voluntariado, envejecimiento activo, personas mayores
\end{abstract}

\section{Leisure, free time and voluntary service in older people}

\begin{abstract}
Many changes are happening at a world scale. Leisure, as social and culture activity does not stay out of this evolution, and at the same time, contributes, from the personal, social economical point of view, to the configuration of certain habits, lifestyle, different ways of understanding leisure and recreational development of new activities. This article proposes the utilization of leisure, free time and voluntary service, within the collectivity of older people, outstanding the importance of their participation on various tasks. This requires a significant dedication of time, knowledge, experience and emotional support, which is not always recognized or appreciated by society. We conclude emphasizing how education, through leisure programs, has modified its conception and is defined trough parameters that refer to autonomy, development, relationship, participation and creativity, also in older people.
\end{abstract}

Key words: Leisure, free time, voluntary service, active ageing, older people

\section{Lazer, recreação e voluntariado em pessoas idosas}

Resumo: Muitas mudanças estão acontecendo em todo o mundo. O lazer, como elemento social e cultural, não fica indiferente a esta evolução e, por sua vez, contribui, do ponto de vista pessoal, social e econômico, com a configuração

\footnotetext{
* Universidad de Granada, Granada, España. Email: imontero@ugr.es

** Universidad de Granada, Granada, España. Email: bedmar@ugr.es
} 
de determinados hábitos, estilos de vida, formas diferentes de entender o descanso e o desenvolvimento de novas atividades. Neste artigo se considera o uso do lazer, o tempo livre e o voluntariado no contexto coletivo de pessoas idosas, destacando a importância da participação deste grupo através de distintas tarefas. Isto requer um investimento significativo de tempo, conhecimentos e apoio afetivo, o que nem sempre é reconhecido e valorizado pelo resto da sociedade. Concluímos destacando como a ação educativa, através de programas de lazer, tem modificado o seu conceito e se define por parâmetros que fazem referencia à autonomia, desenvolvimento, relação, participação e criatividade, também em pessoas idosas.

Palavras-chave: Lazer, tempo livre, voluntariado, envelhecimento ativo, idosos.

Recibido: 14.12.2009

Aceptado: 30.06.2010

$* * *$

\section{Introducción: aspectos socio-demográficos. El envejecimiento de la población}

El envejecimiento de la población es una característica que afecta a la población mundial. Las sociedades envejecen en todas las partes del mundo. Tal como reconoce el Informe 2008 del IMSERSO ${ }^{1}$, los grupos de personas de 65 y más años comienzan a ser un segmento importante de todos los países ${ }^{2}$. No obstante, “envejecer” es algo subjetivo y está condicionado por factores socioculturales, políticos, económicos... Si nos atenemos a los datos puramente cuantitativos, en España, durante los últimos cinco años, la población mayor ha ascendido en más de 250.000 personas, con un incremento del 3,5\%. El panorama demográfico futuro presenta una sociedad envejecida en la que casi un tercio de la población serán personas mayores. En cifras, por cada niño de 0 a 14 años habrá 2,3 personas mayores. En América Latina existe, de forma parecida a Europa, (aunque en distinto grado), un descenso de la población de 15 años, un aumento de la población de 65 años y un engrosamiento de la pirámide entre estas edades $^{3}$. De ello se concluye que la población irá envejeciendo.

\footnotetext{
${ }^{1}$ IMSERSO (Instituto de Mayores y Servicios Sociales) es la entidad gestora de la Seguridad Social en España, destinada a la gestión de los Servicios Sociales complementarios de las prestaciones del Sistema de Seguridad Social y en materia de personas mayores y personas en situación de dependencia.

${ }^{2}$ Datos publicados en el año 2009. "Informe 2008. Las personas mayores en España”. Madrid: Instituto de Mayores y Servicios Sociales (IMSERSO). Disponible en:

http://www.imsersomayores.csic.es/documentos/estadisticas/informe-mayores/2008/volumen-1/00-informe-personas-mayores-2008-vol-01.pdf

3 "La fecundidad en América Latina: un descenso acelerado y heterogéneo con profundas transformaciones demográficas y sociales”. CEPAL (Comisión Económica para América Latina y el Caribe). Observatorio demográfico. $\mathrm{N}^{\circ}$ 5. Abril 2008. Disponible en:

http://redo.me.uk/ndymo/www.eclac.cl/cgi-in/getProd.asp?xml=/publicaciones/xml/8/ 36498/P36498.xml\&xsl=/celade/tpl/p9f.xsl\&base=/tpl/top-bottom.xslt\#
} 
El caso concreto de Chile merece una referencia especial. A diferencia de España y en un país donde la migración internacional no ha sido un factor demográfico importante, los cambios demográficos globales se explican en mayor medida por la evolución del crecimiento natural de la población (natalidad menos mortalidad). La evolución desde niveles altos a bajos de la mortalidad y de la natalidad que se produjo durante el siglo XX, da cuenta del proceso de transición demográfica avanzada en que se encuentra Chile en la actualidad junto a Argentina, Brasil y Costa Rica. Según las proyecciones de población, todo indica que esta tendencia se mantendrá, lo que tendrá múltiples consecuencias demográficas, como el progresivo envejecimiento de la población, e implicaciones sociales, como una disminución de la proporción de población económicamente activa ${ }^{4}$.

Sabemos que el grupo de adultos mayores ha tenido un continuo aumento: superará el 20\% en el año 2025 aproximándose al 30\% hacia el 2050. Este proceso constituye el llamado envejecimiento demográfico de la población. En el índice de Adultos Mayores ${ }^{5}$ (IAM) (expresa el número de adultos mayores por cada cien menores de 15 años), se observa su tendencia a crecer a largo plazo, producto de una esperanza de vida cada vez más elevada junto con el mejoramiento sustantivo de las condiciones de vida y salud. En la actualidad, el sector de adultos mayores sigue siendo "minoría creciente", no obstante su ritmo de crecimiento anual es superior al total de las edades restantes. Se estima que hacia el 2025, en Chile habría 1 adulto mayor por cada menor de 15 años y, hacia el 2050 habría 1,7 adultos mayores por cada menor de 15.

A mediados del presente siglo, Italia, Japón, España y Grecia serían los países más envejecidos ya que, en promedio, los adultos mayores triplicarían a los menores de 15 años. Los 3 primeros países son, en la actualidad, los más envejecidos, con 1,6 adultos mayores por cada menor de 15 años. En otras palabras, el envejecimiento casi se duplicaría en estos países en 43 años más, en tanto que en Chile se triplicaría en igual periodo (INE 2008, op. cit.).

Los datos cuantitativos aportados han venido acompañados de transformaciones socio-económicas, políticas y culturales no menos significativas. A pesar de ello, justo es subrayar que solemos disponer de más cifras sobre el envejecimiento que de serias reflexiones y propuestas formales que respondan a las consecuencias derivadas de estos cambios. En una época centrada en los avances tecnológicos, las personas mayores reman contra corriente ante ideales consagrados de belleza, juventud y poder. Caracteri-

\footnotetext{
${ }^{4}$ Según datos extraídos del siguiente documento: "Población y Sociedad: aspectos demográficos”. Publicado en mayo de 2008 por el INE (Instituto Nacional de Estadísticas). Santiago (Chile). Disponible en: http://www.ine.cl/canales/chile_estadistico/demografia_ y_vitales/demografia/pdf/poblacion_sociedad_enero09.pdf

${ }^{5}$ A lo largo del artículo utilizaremos indistintamente las expresiones "adultos mayores” y "personas mayores" sabiendo que, refiriendo una misma significación, la primera acepción suele ser utilizada en Latinoamérica, mientras que la segunda es más común en los países europeos.
} 
zado por niveles económicos aceptables, sin los problemas de salud de antaño, con 10 ó 15 años de media de vida y con gran cantidad de tiempo libre por ocupar, los mayores quedan socialmente relegados y desplazados en no pocas ocasiones. No obstante, el creciente nivel formativo y cultural de este sector, junto con las importantes experiencias que lo avalan, lo hacen firme candidato a protagonizar actividades y procesos de participación y compromiso socio-comunitario. En España, según diversos autores (Bermejo, Pinazo, Lorente, Limón, Fernández, 2010: 4), estos mayores niveles de autonomía y libertad se manifiestan, entre otros aspectos significativos, en cómo están envejeciendo muchas personas: * Más lentamente, con más capacidades personales, y con más salud; * Con mayor grado de seguridad e independencia económica; * Con mucho tiempo para disfrutarlo; * Optando a la participación activa en equipamientos, instituciones y organizaciones de diverso tipo. Otros expertos hablan de que, efectivamente, España es un "país de viejos"; pero de unos viejos que disponen de más tiempo libre, tienen mejor salud y más educación, un mayor nivel adquisitivo y, por añadidura, otras inquietudes de acuerdo a nuevos estilos de vida (Giró, 2009: 33). Dicho panorama puede generalizarse para muchos de los países europeos. Sin embargo, siendo más precisos, es conveniente señalar que el acceso y las posibilidades se ven limitadas cuando las condiciones económicas, políticas, sociales, que configuran el desarrollo y la calidad de vida de estas personas, no acompañan el proceso. Según informes internacionales ${ }^{6}$, en América Latina aún constan como principales preocupaciones de las personas mayores la falta de seguridad de ingresos (menos del 30\% de latinoamericanos mayores reciben una pensión); la falta de acceso gratuito a los servicios de salud; la participación, dado que aún existe poco reconocimiento, por parte de los gobiernos o la sociedad civil, hacia la implicación activa de las personas mayores en la sociedad; la marginalización, siendo un colectivo especialmente vulnerable a los abusos y violencia. Lo cual significa que son muchas las personas que enfrentan la pobreza conforme envejecen. Y estas condiciones se agravan, aún más si cabe, en el caso de las mujeres mayores.

Efectivamente, cuando hablamos de “adultos mayores" queda claro que no podemos homogeneizarlos o simplificarlos en un determinado colectivo. Las condiciones descritas anteriormente, la variabilidad de los contextos de referencia y actuación, los elementos que, en suma, configuran su vida y desarrollo, requieren, al menos, un acercamiento y un estudio mucho más específico y concreto. En España, uno de los retos que ha de afrontar nuestra sociedad para paliar las desigualdades sociales que aún persisten, son la cobertura de las necesidades asistenciales y económicas de la población jubilada y anciana, la preparación para la jubilación de personas que se encuentran entre los 55 y 65 años y la creación o el desarrollo de recursos para la ocupación del tiempo libre (Hernández Rodríguez, 2009).

\footnotetext{
${ }^{6}$ Consultado en: http://www.helpage.org/es/Global/AmricaLatina-CentrodeDesarrolloRegional/ EnvejeciendoenAmricaLatina
} 
Desde este presupuesto, y aun reconociendo que aún queda mucho camino por recorrer en otros ámbitos, estamos convencidos de que existen maneras diferentes pero posibles de vivir el ocio, tiempo libre y voluntariado, también a estas edades. Queremos resignificarlo de forma expresa.

\section{(Re)significación del ocio y el tiempo libre en la sociedad post-industrial}

En los países de "mayor desarrollo" vivimos inmersos en una sociedad cambiante y dinámica, caracterizada en este nuevo siglo por la productividad y el verdugo del tiempo. Distintas variables resultan afectadas por los cambios que se suceden tan vertiginosamente. El ocio, como elemento social y cultural, no permanece ajeno a tal evolución y, a su vez, contribuye desde el punto de vista personal, social y económico, a la configuración de determinados hábitos, estilos de vida, formas diferentes de entender el descanso y desarrollo recreativo de nuevas actividades. Algunos autores se atreven con el término "ocio posmoderno" para indicar un cambio en la orientación y los significados del fenómeno (Águila, 2007). Relacionando este concepto con los cambios más significativos que se están dando en las sociedades avanzadas (telecomunicaciones digitales inteligentes a gran escala, incremento de la globalización, nuevas formas de poder, cambios en los patrones de consumismo, mayores oportunidades para las mujeres y nuevos contextos de trabajo y vida), parece que una nueva cultura se impone y, por ende, transformaciones en otros órdenes que afectan a la organización, distribución y disfrute de nuestro tiempo.

Subrayamos, antes de seguir avanzando, que hablar de ocio y tiempo libre no es asunto fácil. Implica, no sólo un cuestionamiento de la realidad, sino de los intereses y valores que aportamos de forma implícita cada uno de nosotros cuando ponemos en juego nuestra voluntad de decisión. El primero de estos conceptos, el ocio, es ante todo una vivencia, y como tal una experiencia que, bien entendida, puede ayudar a realizarnos y construir puentes de comunicación hacia los otros, mejorando la comprensión y el entendimiento de nuestro entorno.

Respecto al segundo de los términos enunciados es cierto que, frente a la percepción que en ocasiones podamos apreciar, en muchas sociedades el "tiempo libre" está aumentando y cada vez se le concede mayor interés; no sólo porque se tiende hacia la disminución de las jornadas laborales, sino por el desgraciado fenómeno del desempleo, el desarrollo constante de las nuevas tecnologías que permiten ocupar nuestro tiempo de formas muy diversas, las cada vez más prontas jubilaciones, el mayor nivel formativo, la importancia prestada desde el ámbito legislativo y social... En realidad, no es que ahora dispongamos de más tiempo, sino que su distribución se realiza en función de prioridades muy diferentes. Frente a las sociedades más industrializadas, que entendían el ocio como consecuencia y refuerzo positivo a la actividad central que constituía el mundo del trabajo, 
las sociedades tecnológicas y de consumo actuales lo estudian y ejercen como expresión individual y social, con protagonismo y funcionalidad propios. No obstante, como adelantábamos al inicio de este trabajo, este fenómeno no es generalizable. Por desgracia, en muchos países la realidad viene mostrando que el tiempo libre no está siendo ampliado, y más bien viene siendo reducido en gran escala debido, principalmente, a las condiciones sociales de existencia de la mayoría de las personas que son marcadas por diversas formas de exclusión (Gomes y Elizalde, 2009) y que se extienden, de manera inequívoca, a las personas de más edad. Lo cual, sin duda, constituye también un mecanismo más de marginación desde el no reconocimiento al disfrute de un derecho fundamental.

En otras sociedades, denominadas comúnmente modernas, occidentales, industrializadas, de "bienestar" o "avanzadas", pero donde sigue primando la desigualdad, la posibilidad de disfrutar de ese tiempo libre ha provocado una mayor tolerancia hacia las actividades que se desarrollan con fines más o menos recreativos y lúdicos, pero también una revalorización de la autorrealización e identificación personal a través de expresiones concretas a la hora de elegir cómo organizar el tiempo. Esto último podría reconocerse como la vertiente más "positiva” del fenómeno. En efecto, el ocio, como tal, puede llegar a ser un medio óptimo de compromiso y transformación social, ofreciendo oportunidades de aprender y crecer desde el propio entorno. De ahí surge otro de los grandes conceptos que consideraremos a lo largo de nuestra exposición: el voluntariado. Cada vez hay más evidencia de que el ocio contribuye a la calidad de vida y al desarrollo medio-ambiental a través de las distintas comunidades y naciones (Edginton, 2007).

La finalidad del presente artículo pasa pues por describir tres elementos interrelacionados que adquieren significación propia en un sector concreto de población con protagonismo creciente: las personas mayores. Sin dejar de lado la singularidad y heterogeneidad que provienen de su consideración en un contexto y condiciones de vida específicos, vamos a intentar explicar, a priori, las claves que subyacen tras los términos empleados, al objeto de poder más adelante concretar a qué estamos aludiendo cuando su uso se adecua al colectivo de las personas de más edad.

\section{Concreción de términos: acerca del ocio y el tiempo libre}

Como decíamos, el concepto "ocio" puede tener comprensiones muy diferentes dependiendo del contexto y la sociedad donde se planteen y la finalidad que se persiga. En este sentido, distintas aproximaciones han otorgado comprensiones, en ocasiones, bien distantes. Abordándolo de manera objetiva consiste, ante todo, en aquellos recursos o actividades que, de manera opcional, ocupan nuestro tiempo. Subjetivamente, supone además una experiencia gratificante, que refleja en cierta manera nuestras preferencias y también nuestra forma de ser y manifestarnos libremente. No depende tanto del tiempo empleado o la formación y el nivel adquisitivo 
del que lo experimenta. Es más bien una consecución y expresión de los propios deseos y sentimientos que laten tras un mundo deseado (Cuenca, 2004). En la misma línea se expresa Gil Calvo (1995), al señalar que el ocio ha de sustentarse en dos supuestos básicos; por un parte, lo que se hace en ese tiempo debe implicar actividades voluntarias, libremente elegidas y no impuestas por ningún estado de necesidad; por otra, que no sean actividades lucrativas, independientes de cualquier clase de retribución diferida, pues su única recompensa inmediata ha de ser la misma participación.

Llegados a este punto, proponer una definición acerca del ocio nos obliga a recoger conceptos y revelar intereses y valores que, en muchas ocasiones, quedan ocultos tras diferentes posicionamientos. Probablemente cada uno de nosotros podría añadir elementos que confieren un carácter propio a la revalorización personal de tal experiencia. Lo evidente hasta ahora es que es un derecho, sin distinción de sexo, procedencia y por supuesto edad, que va más allá del mero divertimento y que se relaciona con formas diversas de entender nuestro tiempo.

Expertos que han estudiado el tema de forma rigurosa enfatizan algunas características sobre el ocio. El profesor Cuenca las presenta en una definición de Kriekemans: "El ocio es una recreación, o sea, un medio para restablecer la voluntad y el valor de vivir” (Cuenca, 1999: 20). Destaca el término recreación como experiencia básica de reconocimiento personal y sustento de los valores. Desde este presupuesto no sólo alude a la posibilidad del disfrute y el deleite en la consecución de determinadas actividades, sino que provoca una felicidad y satisfacción compartidas. En un fin en sí mismo. La misma perspectiva es resaltada por uno de los autores más relevantes en este campo. El profesor Dumazedier $(1968)$ destacaba las tres " $D$ " en su estudio: descanso, diversión y desarrollo, incorporando la creatividad como elemento constitutivo de la propia personalidad. Otros expertos se expresan a través del término loisir en plural ${ }^{7}$ para referirse a un cierto número de representaciones de actividades concretas que cada uno puede escoger libremente sin limitación alguna, fuera del tiempo consagrado a las ocupaciones profesionales en sentido estricto, aun reconociendo la confusión que puede derivarse de tal multiplicidad en las actividades como de la ambigüedad de la misma noción de trabajo (Leif, 1992: 32-33).

Para Csikszentmihalyi (2001, 2004), el ocio es una de las condiciones fundamentales para disfrutar la vida. Sin embargo, considera que existe un problema esencial en las sociedades actuales dado que, en muchas ocasiones, no tenemos las destrezas incorporadas para utilizar el tiempo libre de forma satisfactoria, lo que puede derivar en el "ocio peligroso". La importancia del ocio, desde el punto de vista del desarrollo humano, está en que ofrece oportunidades para salir de la rutina y experimentar y, por otro

\footnotetext{
${ }^{7}$ En francés, suele hablarse de loisirs para referirse a los diversos modos de emplear el tiempo de ocio, en vez de utilizar sólo el término loisir, en singular. En castellano, en cambio, no existe el plural, por ello se mantiene el término en su acepción original.
} 
lado, en su capacidad de potenciar las habilidades que pueden ayudarnos a descubrir la dimensión lúdica de todo lo que cotidianamente hacemos.

¿Cuáles son, entonces, las notas que diferencian los términos ocio y tiempo libre? Cuando hablamos de éste, solemos referirnos a un tiempo posible cuyo empleo depende, en cierta medida, de nuestra propia elección. Es algo objetivo, medible y cuantificable. Lo constituyen todos aquellos momentos en los cuales la persona está fuera del trabajo y, por ende, no siempre equivale a tiempo disponible para el ocio o la recreación (Elizalde, 2010). Por tanto, el tiempo libre sería el “continente”, mientras que el ocio sería el "contenido", de tal forma que llegamos al ocio cuando empleamos el tiempo libre realizando aquello que nos gusta y procura nuestro recreo y cultivo.

Lo que sí cabría destacar en este sentido, es que el desarrollo del tiempo libre, tal como señala la Carta Internacional de WLRA para la educación del tiempo libre ${ }^{8}$, es facilitado por el suministro de condiciones de vida básicas, tales como seguridad, vivienda, alimentación, ingresos, educación, recursos, equidad y justicia social. De no cumplirse estas mínimas condiciones sería paradójico hablar del disfrute en el tiempo libre. En todo caso, la educación del tiempo libre debe adaptarse a las necesidades y requerimientos locales de países y regiones diferentes, tomando en consideración los diferentes sistemas sociales, culturales y económicos.

Tras lo expuesto, ¿¿cómo podría ser entendido el ocio? Suele concebirse como una experiencia más personal relacionada con intereses y deseos, en la que es muy difícil determinar el tiempo de proyección y deleite. Por eso es, además, una vivencia dinámica y creativa.

La Carta Internacional para la Educación del Ocio, resultante del Seminario de WRLA celebrado en Jerusalén en 1993, es uno de los documentos más representativos que incluye los avances logrados en este campo. En esta carta el ocio se entiende como "área específica de la experiencia humana, con sus beneficios propios, entre ellos la libertad de elección, creatividad, satisfacción, disfrute y placer, y una mayor felicidad. Comprende formas de expresión o actividad amplias cuyos elementos son frecuentemente tanto de naturaleza física como intelectual, social, artística o espiritual”. Así pues constituye un derecho humano, un recurso de salud y signo de calidad de vida, una acción voluntaria elegida en función de la

\footnotetext{
${ }^{8}$ WLRA (World Leisure and Recreation Association), "International Charter for Leisure Education”, en ELRA (European Leisure and Recreation Association), Summer, 1994, pp. 13-16.

Fundada en 1952, ha pasado a denominarse Organización Mundial de Ocio (World Leisure Organization). Es una asociación no gubernamental a nivel mundial, de la cual forman parte personas y organizaciones dedicadas a descubrir y fomentar las condiciones que permitan un mejor ocio. El WLO promueve el ocio como parte integral, cultural, económica, social y el desarrollo sostenible del medio ambiente.
} 
satisfacción que pudiera proporcionarnos de manera íntima o en comunidad. Por otro lado, la experiencia comunitaria del ocio permite relacionar los propios intereses con el resto del grupo y amplía los lazos afectivos entre los que comparten esa misma visión de la realidad.

En referencia a su ejercicio o puesta en marcha, podemos subrayar algunas dimensiones que, desde ámbitos complementarios, permiten explicar y planificar diferentes expresiones. Estas expresiones aparecen relacionadas con distintos modos de vivir el tiempo libre, ámbitos, ambientes, equipamientos y recursos. Pero de ninguna manera se excluyen, sino que contribuyen a comprender y organizar la realidad del desarrollo humano. Cuenca $(1995,1999,2004)$ recoge cinco dimensiones fundamentales que vamos a tratar de sintetizar brevemente: * Dimensión lúdica: referida al modo en que vivimos y asumimos el tema del disfrute y el juego, las relaciones que se establecen, los intereses subyacentes... Tiene pues, una finalidad de descanso y diversión, necesaria para el equilibrio físico y psíquico. * Dimensión creativa: relacionada con el capital cultural de las personas y grupos, es un disfrute más ligado a procesos de reflexión. Nos ayuda también a adquirir los saberes desde una vertiente no productiva y a afirmar así nuestra identidad personal a través de un proceso de autorrealización.* Dimensión ambiental-ecológica: expresa la sensibilidad hacia el impacto que ejercen las actividades de ocio y ayuda a planificar un desarrollo sostenible que respete la armonía medioambiental. Tiene que ver con el entorno físico y urbano de la comunidad, pero también con la vivencia de ocio unida a la naturaleza. * Dimensión festiva: permite llevar a cabo experiencias que cohesionan a la comunidad a través de determinadas vivencias. La fiesta se entiende como manifestación extraordinaria de ocio a través de una exaltación de valores como la libertad, la solidaridad, la cohesión y la identidad. * Dimensión solidaria: como signo de calidad humana y sensibilidad desde una vivencia altruista y social. Se centra sobre todo en la apertura, la comunicación y la entrega al otro, la ayuda desinteresada, el compromiso... que repercuten en el desarrollo comunitario. En esta dimensión cabe hablar, como veremos, del voluntariado.

Como decíamos, aun reconociendo que unas u otras puedan aparecer con mayor frecuencia, estas dimensiones suelen entrelazarse, de manera que rara vez es posible aislarlas en la realidad. A lo que sí contribuyen es a diagnosticar y planificar mejor los procesos que ponemos en juego para llevar a cabo un óptimo aprovechamiento del tiempo y, como consecuencia, un buen planteamiento de nuestro tiempo de ocio. Pero, ¿̇ealmente son elementos comunes? O, dicho de otro modo, tales dimensiones, ¿podrían considerarse presentes, en cualquier caso, en la práctica del ocio? En efecto, esta conceptualización y su práctica podría evidenciarse en sociedades que así lo permiten. Como decíamos, en excesivas ocasiones no es fácilmente visible, en tanto en cuanto existen aún amplios sectores de población excluidos de la posibilidad de ejercer y satisfacer otras prioridades básicas y fundamentales. Aun así, creemos firmemente que el ocio, entendido como experiencia esencialmente humana y socializadora, sigue siendo una de las necesidades a cubrir en todos los países y sociedades. Si bien es cierto que 
las actuales prácticas de sobreconsumo y homogeneización pueden socavar las posibilidades y disfrute de nuestro tiempo, se requiere aún más si cabe una atención mayor en este sentido. Estamos de acuerdo con otros autores (Elizalde, 2010) en que actitudes críticas, creativas y liberadoras, pueden colaborar en la transformación social al objeto de construir sociedades más solidarias, justas y sustentables. Dada la posibilidad y partiendo de esta libre elección, en adelante se propone el envejecimiento activo como principio y concepto emergente en torno al cual poder centrar los elementos claves de participación e implicación social en países en los que ya existen alternativas en esta dirección.

\section{El envejecimiento activo, principio clave en la participación social}

Retomando el discurso con el que comenzábamos, es en los países más occidentales donde, en los últimos años, el uso del tiempo entre las personas mayores está experimentando cambios notables. Por ello desde instancias sociales y políticas está resultando de gran trascendencia el acercamiento a los intereses, motivaciones, demandas y deseos de este sector de población, puesto que gran parte del desarrollo y el funcionamiento de nuestra sociedad dependen directamente de las actividades que todos realizamos en el vivir de cada día. Del mismo modo, el empleo del tiempo puede ser considerado un indicador de la calidad de vida de las personas. De manera que las sociedades, en última instancia, también configuran, posibilitan, fomentan, elementos y espacios para el uso y disfrute de los diferentes tiempos y modos de vida. Las últimas investigaciones realizadas en cuanto al uso del tiempo en personas mayores en España ${ }^{9}$, arrojan datos e informaciones realmente significativas. En una sociedad donde el "mercantilismo" y la productividad suelen imperar y ganar terreno, pocas veces se tienen en cuenta las actividades que se realizan fuera del mercado de trabajo. Sin embargo, las personas mayores contribuyen al sostenimiento del Estado de Bienestar desde su apoyo a la familia con el cuidado de nietos, de personas dependientes y ayudas informales a otros hogares. Del mismo modo, su participación ciudadana a través de tareas de voluntariado (entre otras) supone una importante inversión de tiempo, energía, conocimientos, apoyo afectivo, que no siempre es reconocido ni valorado por el resto de la sociedad. Autores como Belsky (1996) señalan que, para disfrutar de una vejez feliz y plena, tan importante es estar razonablemente sanos y bien adaptados, como encontrar el estilo de vida que mejor exprese nuestro "yo interno".

En la actualidad se habla de tres tipos de envejecimiento que no siempre son justamente diferenciados: satisfactorio, productivo y activo.

\footnotetext{
${ }^{9}$ En el Boletín sobre el Envejecimiento dependiente del IMSERSO (Instituto de Mayores y Servicios Sociales) en España, ${ }^{\circ} 27$ de marzo (2007), se publica un interesante artículo al respecto: "Uso del tiempo entre las personas mayores".
} 
Así, el envejecimiento satisfactorio es el preocupado por la mejora de los aspectos físicos o fisiológicos, como evitar enfermedades y discapacidades, mantener un buen funcionamiento físico y cognitivo y sentirse vivo. Otro concepto relacionado es el de envejecimiento productivo. Este término es relativamente reciente (nació en la década de los años ochenta) y, más allá del sentido estrictamente económico que parece conllevar, alude a las "actividades significativas realizadas por personas mayores, mediante un trabajo remunerado o no, que contribuya a la mejora del bienestar social y a la producción o desarrollo de bienes y servicios”10. Se contradice con la visión negativa relacionada con el menoscabo del potencial a ciertas edades. Por su parte, la Organización Mundial de la Salud (OMS) utiliza el término envejecimiento activo para expresar el proceso por el que se consigue este objetivo: "el envejecimiento activo es el proceso por el cual se optimizan las oportunidades de bienestar físico, social y mental durante toda la vida con el objetivo de ampliar la esperanza de vida saludable, la productividad y la calidad de vida en la vejez”. El término fue adoptado por esta organización a finales del siglo XX con la intención de transmitir un mensaje más completo que el de envejecimiento saludable y reconocer los factores, además de la mera atención sanitaria, que afectan a cómo envejecen individuos y poblaciones. Uno de sus objetivos básicos es: "Promover la autonomía y la participación plena y activa de las personas mayores en la comunidad, en base a los principios del Envejecimiento Activo"11.

En este sentido, en España, algunos de los espacios con creciente protagonismo y repercusión socio-educativa lo constituyen los “centros de día”. De manera emergente van tomando mayor trascendencia como centros de promoción del bienestar entre las personas mayores. Tendentes al fomento de la convivencia, la integración, la participación, la solidaridad y la relación con el medio social, sirven de encuentro para el desarrollo de distintos tipos de actividades. Entre sus objetivos ${ }^{12}:{ }^{*}$ Facilitar una mayor

\footnotetext{
${ }^{10}$ Definiciones extraídas de los apuntes correspondientes al Curso Experto Universitario en Gerontagogía: intervención socioeducativa con personas mayores, celebrado en Granada ( $2^{a}$ edición, 2001). Elaboración del módulo por parte del profesor Mariano Sánchez Martínez.

${ }^{11}$ Según IMSERSO (2007), se entiende por envejecimiento activo la forma de afrontar esta etapa vital, potenciando la autonomía (física y mental), el crecimiento personal, las relaciones interpersonales (intergeneracionales) y la participación social. (II Jornadas sobre Discapacidad y Envejecimiento Activo. Buenas Prácticas. Madrid, septiembre 2007). Por su parte, la OMS (2002, p. 79 y ss), lo define así: "El envejecimiento activo es el proceso de optimización de las oportunidades de salud, participación y seguridad con el fin de mejorar la calidad de vida a medida que las personas envejecen...” Desde una perspectiva política, “... se basa en el reconocimiento de los derechos humanos de las personas mayores y en los Principios de las Naciones Unidas de independencia, participación, dignidad, asistencia y realización de los propios deseos... Y respalda su responsabilidad para ejercer su participación en el proceso político y en otros aspectos de la vida comunitaria” (OMS (2002) Trad. por Regalado, P. "Envejecimiento Activo: un marco político”. En Revista Española de Geriatría y Gerontología 2002, 37 (S2), 74-105).

${ }^{12}$ Recogidos del Reglamento de los Centros de Día, Granada, diecinueve de mayo de 2003.
} 
cohesión entre la población mayor. * Fomentar la integración en la comunidad donde se insertan, a través de la realización de actividades conjuntas.* Contribuir a la prevención del aislamiento de la población de la Tercera Edad mediante la realización de actividades con otros grupos e instituciones, llevando a cabo acciones de intercambio y ayuda mutua. Teniendo estos elementos en cuenta, existirán actividades adecuadas para ocupar el ocio y tiempo libre, estimular la participación y promover la cultura. De forma más concreta, irán dirigidas a enriquecer la personalidad, fomentar la convivencia y solidaridad y potenciar actividades de integración intergeneracional.

Tal como venimos exponiendo, consideramos que lo esencial, independientemente del tipo de actividad y del centro donde se lleve a cabo, es que la elección adoptada sea libre y conforme a los centros de interés de cada individuo, produzca placer y bienestar individual y colectivo, y llegue a convertirse en fuente de experiencias personales y grupales a través de la cual poder expresar los propios deseos y potencialidades. En todo caso, el uso del ocio y tiempo libre ha de ofrecer al sujeto la oportunidad de disfrutar y desarrollarse desde la alternativa que, de acuerdo a sí mismo, manifieste o prefiera. Estamos de acuerdo en que el ocio puede representar tanto un espacio de libertad y dignificación, como una forma de reforzar las injusticias y opresiones sociales (Gomes y Elizalde, 2009). Pero del mismo modo creemos que, al menos en las sociedades con mayores posibilidades, también puede llegar a ser vehículo de transformación social y objeto de educación al reflexionar sobre la propia sociedad y los elementos óptimos y alienantes que la configuran. Esto, de alguna forma, repercutiría y contribuiría a una mayor conciencia desde el ejercicio de una ciudadanía más responsable. Contribución, sin duda, tan realizable como necesaria. Parece evidente que la educación en las sociedades democráticas requiere el desarrollo de un tipo de ciudadanía comprometida, no sólo con el mantenimiento de la "anatomía democrática de la comunidad”, sino también dispuesta a implicarse cotidianamente en hacer democrática la convivencia social (Martín, 2006: 79). Aun así, reconocemos que abordar el papel de la educación implica definir tanto los conocimientos y las capacidades que exige la formación de todo ciudadano, como la forma institucional a través de la cual ese proceso de formación debe tener lugar (Fernández, 2001). Desgraciadamente, en este sentido, las alternativas no siempre se garantizan. Con la pretensión de concretar las experiencias más cercanas a nuestro objeto de estudio, y centrándonos en las sociedades que, al menos, presumen de tales posibilidades, vamos a ver cómo ocupan su tiempo las personas mayores en Europa y las condiciones y variables que inciden en tal elección.

\section{Ocupación del tiempo libre en el colectivo de personas mayores en Europa: variables incidentes}

Comenzamos reconociendo que no existen aún demasiados trabajos e investigaciones que centren sus objetivos en delimitar cómo los adultos mayores se manifiestan y entienden el uso y disfrute del ocio y tiempo 
libre. Quizá por esa comprensión equivocada de que, quien está jubilado, no tiene otra cosa que hacer que "disfrutar" del tiempo sin demasiadas exigencias ni justificaciones previas. Sin embargo, aun reconociendo las circunstancias específicas y concretas que ayudan a interpretar la organización del tiempo en cada población y sociedad de forma tan desigual, es posible intentar un acercamiento desde las distintas dimensiones que describíamos en el apartado anterior. Estas dimensiones podrían expresarse en relación a determinadas actividades que el profesor Castro (1990: 60), recoge en cuatro grandes apartados: * Actividades con algún componente físico: deportes, paseos, viajes, excursiones...; * Actividades de tipo práctico: bricolaje, trabajos manuales, artesanía...; * Actividades culturales: lectura, música, teatro, cine, radio, televisión..; * Actividades de carácter social: tertulias, fiestas, actividades organizadas por clubes, asociaciones...

En cuanto al tiempo efectivamente empleado en estas ocupaciones, tomando como referentes las cifras correspondientes a la población identificada, según los últimos datos del Informe del IMSERSO, año 2006, en España, entre los mayores ${ }^{13}$, más de la mitad del horario que queda, una vez satisfechas las necesidades fisiológicas y de cuidado personal, es tiempo libre que se dedica al ocio y las relaciones personales. Por término medio, los mayores de los catorce países ${ }^{14}$ invierten en estas actividades unas seis horas y media, casi tres más que el conjunto de la población de 20 a 74 años que se utiliza con fines comparativos. Como decíamos, hasta cierto punto este concepto de uso del tiempo se define de manera residual, es lo que queda una vez se han asumido las obligaciones que impone la vida productiva y reproductiva; ese carácter tiene su reflejo en las diferencias entre hombres y mujeres (las mujeres disfrutan de menos tiempo de ocio) y, en alguna medida, en la distribución precisa de ese tiempo en las distintas tareas. Casi la mitad, unas tres horas, se dedica a la audiencia de televisión y casi una hora más al ocio pasivo, es decir, a descansar sin hacer nada en particular. Sin embargo, tomando el sector de población juvenil como referencia, los mayores no son muy distintos: en el grupo de edades de 20 a 74 años, también la mitad del tiempo libre se invierte en audiencia de televisión; los más jóvenes ven la televisión durante menos tiempo que los mayores (unas dos horas), pero el tiempo que le dedican es casi la mitad de todo el tiempo libre del que disponen. En el resto de las actividades las diferencias son más sutiles: los mayores leen más y oyen más la radio, sobre todo los varones, y participan más en actividades religiosas, en este caso sobre todo las mujeres.

En tal sentido, las diferencias de género son constatables, sobre todo en aquellas actividades que tienen que ver con el hecho de salir fuera

\footnotetext{
${ }^{13}$ Según este organismo, se considera población de edad o mayor a aquellas personas que tienen 65 años o más; el umbral es arbitrario, pero generalmente aceptado. Naciones Unidas también considera el umbral de los 60 años, población mayor.

${ }^{14}$ El IMSERSO ha considerado los siguientes 14 países para los datos que se refieren dentro del Informe 2006: Alemania, Bélgica, Eslovenia, España, Estonia, Finlandia, Francia, Hungría, Italia, Letonia, Lituania, Polonia, Reino Unido y Suecia.
} 
de casa, como la asistencia a clubes, asociaciones o centros de mayores. Normalmente esto obedece a las representaciones sociales que perpetúan comportamientos estereotipados también en el colectivo de las personas de más edad: la mujer suele estar vinculada al hogar y el hombre con espacios fuera de éste. También la edad influye, puesto que al aumentar suele deteriorarse la salud y disminuye la actividad en todos los ámbitos. Sin embargo, la mujer está más comprometida con las actividades culturales (los Centros de Adultos y las Universidades de Mayores tienen una proporción mucho mayor de población femenina).

Siguiendo la comparativa con los jóvenes, los mayores pasan menos tiempo delante del ordenador, aunque esta actividad va adquiriendo cierta presencia en sus vidas cotidianas, sobre todo en las de los varones. En otros capítulos, el tiempo de los mayores se ocupa de una forma bastante similar al conjunto de la población, por ejemplo, invierten poco tiempo en cultura y espectáculos, pero tampoco los más jóvenes dedican mucho tiempo a estas prácticas. Algo parecido sucede con el ejercicio físico, los mayores practican algo más; eso sí, el de los jóvenes suele centrarse en deportes propiamente dichos, mientras que los mayores acostumbran, sobre todo, a pasear. Los varones mayores dedican también más tiempo a lo que EUROSTAT ${ }^{15}$ llama “ejercicio productivo” que incluye actividades como la caza y la pesca. La comparación con los mayores españoles indica que, en España, los hombres mayores tienen más tiempo de ocio que el conjunto de los europeos; el resultado de esta diferencia es un aumento de las diferencias de género y de edad en las formas de utilizar el tiempo. Lo que sucede es que los mayores españoles participan menos que los de otros países en el trabajo doméstico, lo que les deja más tiempo libre. Además, dedican considerablemente más tiempo a los paseos, seguramente en correspondencia con las condiciones climáticas del país; sin embargo leen menos y dedican menos tiempo también a oír música o las emisiones de radio. Conviene destacar que la Comisión Europea considera el envejecimiento como una de las diez áreas relevantes en el mantenimiento del crecimiento sostenible. Vemos, por tanto, que las personas mayores dedican una alta proporción de su tiempo al disfrute de aquellas actividades que más les satisfacen y, en buena parte, pueden ser compartidas con otras.

En definitiva, se constata que, en la actualidad, la acción educativa a través de programas de ocio ha modificado su concepción y se define a través de parámetros que hacen referencia a la autonomía, desarrollo, relación, participación, convivencia, creatividad y utilidad ${ }^{16}$ (Limón, 2004). Consecuentemente, tal como expresa la profesora Núñez, "ha llegado el

\footnotetext{
${ }^{15}$ El Eurostat (Statistical Office of the European Communities) es la oficina estadística de la Comisión Europea que produce datos sobre la Unión Europea y promueve la armonización de los métodos estadísticos de los estados miembros.

${ }^{16} \mathrm{M}^{\mathrm{a}} \mathrm{R}$. Limón (2004: 165 y ss) elabora un capítulo al respecto muy interesante, en donde pone de relieve la importancia del ocio y tiempo libre a estas edades y, en concreto, el papel que pueden desempeñar los juegos de cartas como espacio de encuentro y desarrollo entre los mayores.
} 
momento de volver a reivindicar la educación como tiempo de ocio en su sentido etimológico: la educación como oferta democrática de igualdad de oportunidades de acceso a la cultura amplia, plural, que permita el enriquecimiento intelectual de todos” (Núñez, 2003: 47). Es preciso entonces, que las normativas faciliten la no discriminación y promuevan la inclusión efectiva. Consideramos, desde esta perspectiva, que la acción del voluntariado es una expresión real, también en el caso de las personas mayores, para contribuir al ejercicio y derecho de mayores niveles de equidad, equilibrio e inclusión social. Y que este ejercicio es válido y realizable en cualquier comunidad con un mínimo de inquietud y responsabilidad ética y social.

\section{El voluntariado: expresión solidaria en las personas mayores}

El voluntariado es una de las actividades que, actualmente, está ocupando un creciente terreno entre el sector de población mayor perteneciente a las sociedades occidentales más avanzadas. Sabemos que con la jubilación, las perspectivas, motivaciones y la manera de organizar el tiempo pasan a modificarse sensiblemente. Es entonces cuando se dejan a un lado las obligaciones impuestas por el mundo laboral y se ejerce más que nunca la "opcionalidad” y una exaltación del tiempo disponible. Por otro lado, en los países de mayor desarrollo, se constata que se accede a la jubilación, no sólo con mejores recursos materiales, económicos y sociales que las generaciones precedentes, sino con más deseos y oportunidades de realizar actividades de ocio (Bazo, 1999).

Efectivamente, uno de los principales fundamentos del envejecimiento activo es la participación social y ciudadana de los individuos, es decir, que las personas mayores sean partícipes de su entorno. En este contexto surge el voluntariado, refiriendo todas aquellas tareas que, sin retribución económica, son realizadas por el bien de la comunidad (Del Barrio, 2007). Así, en los últimos años el voluntariado ha experimentado un gran auge entre las personas mayores que, además de atender sus obligaciones, dedican su tiempo de modo continuo y desinteresado a favor de los más desfavorecidos.

Hasta fechas muy recientes, las asociaciones sólo se ocupaban de la asistencia a este colectivo, e incluso mostraban una falta de interés por incorporar a personas mayores como voluntarios ya que suponían desmotivación por participar en una organización. En la actualidad, cada vez más, se preocupan por hacerlos participar activamente, no sólo por la importancia dada a que sean protagonistas de su propio desarrollo, sino por la enorme cantera de recursos humanos con una amplia disponibilidad horaria, que puede cubrir horarios y tareas donde es difícil encontrar otros recursos.

En todo caso, ¿a qué hacemos referencia cuando hablamos de “voluntariado”? Como tantos otros términos que se utilizan con excesiva 
frecuencia y versatilidad, el voluntariado es un concepto cargado de connotaciones y acepciones no siempre exactas. Según la Ley de voluntariado de 15 de enero de $1996^{17}$ puede definirse como "el conjunto de actividades de interés general, desarrolladas por personas físicas, siempre que las mismas no se realicen en virtud de una relación laboral, funcionarial, mercantil o cualquier otra retribuida y reúna los siguientes requisitos: * Que tengan carácter altruista y solidario; * Que su realización sea libre, sin que tengan su causa en una obligación personal o deber jurídico; * Que se lleven a cabo sin contraprestación económica, sin perjuicio del derecho al reembolso de los gastos que el desempeño de la actividad voluntaria ocasione; * Que se desarrollen a través de organizaciones privadas o públicas y con arreglo a programas y proyectos concretos”.

Dicho esto, las actividades de voluntariado se sitúan a caballo entre las actividades no remuneradas (porque también podrían constituir un empleo), las de ocio (porque son elegidas voluntariamente y ocupan el tiempo libre después de las obligaciones) y las actividades sociales (fuera del ámbito familiar). La acción voluntaria es una acción dirigida al desarrollo de la comunidad. Desarrollo basado en la participación de personas, de grupos, de la comunidad en la resolución de sus problemas.

En realidad, el voluntariado, entendido como fenómeno participativo y regulado en el caso de este colectivo, es un hecho relativamente reciente. Lo cual contribuye a que, con frecuencia, sean confusos los datos que manejamos acerca del fenómeno. Podría decirse que muchas personas mayores son voluntarias "sin saberlo" y, por tanto, es improbable que tengan conciencia como tales y que se ubiquen formal e institucionalmente en el marco normativo que hoy se ofrece al voluntariado. En efecto, la autoconciencia de las personas mayores como voluntarias y su incorporación a los referidos marcos normativos está siendo, sin duda, creciente en el caso de personas que colaboran en actividades de voluntariado al servicio de terceras personas, sea en organizaciones que cuentan con voluntarias y voluntarios de diferentes edades, sea en organizaciones en las que las voluntarias y voluntarios son, exclusiva o preferentemente, personas mayores. Sea como fuere, parece existir un consenso bastante extendido entre las personas expertas y estudiosas del voluntariado en los países del entorno europeo acerca de que se trata de un fenómeno en auge o crecimiento desde el punto de vista cuantitativo (Fantova, 2002). Sin embargo, las últimas cifras hablan de que, en España, tan sólo un 0,6\% de personas mayores trabaja al servicio de una organización, el mismo porcentaje que el correspondiente a las personas de entre 25 y 44 años. El trabajo voluntario parece estar poco extendido y el número de personas que participa en estas actividades es aún relativamente bajo (Del Barrio, 2007). A pesar de ello, las personas mayores voluntarias se comprometen mucho más que otros grupos de edad. Esta conducta más comprometida se atribuye al hecho de que el voluntariado resulta ser especialmente útil para las personas mayores. Su

${ }^{17}$ Publicada en España. BOE (Boletín Oficial del Estado) nº 15, de 17 de enero de 1996. 
naturaleza productiva tiene un efecto positivo sobre varias dimensiones del bienestar, tales como la satisfacción de vida o la salud. Diversos estudios han demostrado que el adecuado empleo del ocio de las personas mayores influye más sobre la percepción de satisfacción en la vida que otros factores (salud, ingresos, educación, trabajos anteriores. etc.) (Meléndez, 1991). Otras investigaciones han destacado una fuerte relación entre voluntariado y satisfacción en la vida. Esta relación se mantiene incluso considerando factores tales como edad, percepción de la salud, y nivel socioeconómico (Maizel, 1987). Del mismo modo, estudios acerca de la conexión entre voluntariado y salud en personas mayores, se han centrado en múltiples aspectos que manejan variables como la salud física, el bienestar psicológico y la longevidad (Dávila y Díaz, 2009).

Efectivamente, según estudios recientes (Erlinghagen y Hank, 2005), la edad, la educación, la salud y la implicación en otras actividades sociales, tienen una gran importancia en la tendencia de los individuos a dedicarse al voluntariado. Las conclusiones extraídas ponen de manifiesto la necesidad de mantener una perspectiva contextual respecto al voluntariado, y explican el papel de las instituciones y de la cultura, así como de las políticas y los programas elaborados para favorecer que los ciudadanos de más edad hagan uso de su potencial productivo en beneficio de ellos mismos y de la sociedad. Siguiendo los datos extraídos del estudio citado (op. cit. 2005), más allá de todas las diferencias transnacionales hay una proporción importante, de hasta el 20 por ciento, de la población europea de 50 o más años de edad, que se dedica al trabajo de voluntario y su potencial productivo ni siquiera se podría valorar todavía en toda su posible extensión.

Un aspecto importante de los señalados destaca lo decisivo de tener siempre presente el aspecto beneficioso de esta actividad para quienes son voluntarios: las personas mayores que participan no lo hacen sólo en beneficio de otros, sino que ellas, por sí mismas, experimentarán una mayor calidad de vida a través de su participación activa en la sociedad. En esta línea, el voluntariado no debe contemplarse aisladamente del contexto social más amplio en el que tiene lugar. Como fenómeno cultural y económico, el voluntariado es parte de la forma en que las sociedades se organizan, asignan responsabilidades sociales y esperan compromiso y participación por parte de los ciudadanos. Quizá por ello sea necesario hacer hincapié en la necesidad de mantener una perspectiva contextual en los análisis sobre el voluntariado, así como en las políticas y en los programas creados para potenciar que los ciudadanos y ciudadanas de más edad hagan uso de su capacidad productiva en beneficio de sí mismos y de la sociedad. Es un ejercicio crítico que posibilita, al mismo tiempo, una nueva perspectiva frente al estilo de vida actual. De este modo, convenimos con otros autores (Gomes y Elizalde, 2009; Elizalde, 2010), en que experimentando el ocio con una actitud contrahegemónica frente al modelo social y cultural actual, excluyente por otra parte para amplios sectores de la población, se contribuye a transformar positivamente no sólo el entorno al cual pertenecemos, sino la conciencia y el pensamiento que pueden favorecer un mayor compromiso en el ejercicio de crecientes niveles de equilibrio y equidad social. 


\section{Recapitulando: el método interactivo como propuesta de implicación socio-educativa}

En este punto cabría preguntarse: ¿qué papel en entonces el que le corresponde a las personas mayores, siempre que los condicionantes sociohistóricos, políticos y económicos así lo permitan, cara a la efectiva implicación y participación socio-comunitaria? Si planteamos la respuesta desde la educación, por otra parte nuestro ámbito de estudio, corrobora el profesor García Mínguez: "la clave de la autoconciencia está en concitar el potencial de la experiencia cara a un acontecer duradero mediante la educación como instrumento de cambio y transformación” (2004: 143).

Siguiendo el hilo conductor de lo que venimos exponiendo, lo cierto es que la inversión en el capital humano es fundamental para captar la esencia de los procesos socioeducativos implicados en el devenir social. Si partimos de contextos favorecedores, las personas mayores son, al igual que el resto de los participantes, miembros de pleno derecho ante las posibilidades de actuación e implicación socio-comunitaria. Desterrar ideas benefactoras que no hacen sino justificar situaciones con fundamentación y legitimidad más que probadas, es una cuestión que habrá que encarar seriamente también desde la participación social.

Retomando las filosofías que argumentan dicha participación, queda suficientemente probado el hecho de que la sociedad no es puro instrumento reproductor, sino aparejo en evolución constante representado por los sujetos que lo dotan de significado. O lo que es lo mismo, la propia implicación social ofrece soluciones a determinadas cuestiones en función de las representaciones o concepciones, imágenes e ideas que circulan en la sociedad y que caracterizan dando forma a los grupos que la integran.

Efectivamente, el sector de las personas de edad provecta es significativamente susceptible de "soportar" ciertos estereotipos y percepciones erróneas y contaminantes de aquellas posibilidades y potencialidades que pueden llegar a desarrollar en la sociedad. Sin embargo, en un análisis más detenido acerca de tales interpretaciones, cuando además de abarcar los significados literales pensamos en las impresiones y reacciones de la gente acerca de los mismos, entramos en la dinámica del conocimiento sobre el pensamiento, la actitud y los sentimientos y emociones de los que participan en tales discursos. Es así como llegamos a un entendimiento global y más cercano a la realidad que queremos descubrir (Montero, 2003).

Si, tal como venimos defendiendo, pretendemos movilizar y potenciar en personas y grupos una comprensión y una serie de capacidades que, en definitiva, permitan optimizar los contextos donde se desenvuelven, las personas mayores son dignos y expresos destinatarios también de la implicación socio-comunitaria. En efecto, los principios de actividad, independencia y participación en los que se centra la implicación de las personas 
de más edad, toman un cariz específico a partir de la perspectiva transformadora que acometemos.

Apreciando no tanto lo que la persona es, sino aquello que puede llegar a ser, (principio de actividad), se permite rescatar en el mayor competencias que conectan sus intereses y necesidades con la misma vida. Es esto lo que, en buena medida, le posibilita avanzar hacia actitudes con un creciente compromiso social y, sin lugar a dudas, de manera más enérgica y optimista en el acercamiento a su realidad.

Como ya revelábamos, distintas investigaciones han demostrado que la actividad recreativa y de ocio es la que más influye en la satisfacción de vida y el bienestar físico de los jubilados (Csikszentmihalyi, Cuenca, 2001). De lo que se trata es de adecuar alternativas a los propios intereses, posibilidades y capacidades. De ahí que el segundo principio, el de la participación, vaya encaminado a fomentar vías y caminos que permitan desarrollar, en efecto, las capacidades de relación interpersonal y el ejercicio creativo que acompaña a toda acción socio-cultural. Aprovechar las oportunidades que, sin duda, cada vez se brindan con mayor frecuencia a las personas de edad avanzada desde instancias particulares y gubernamentales, pero también, por qué no, provocar que sean ellas mismas quienes decidan de qué manera desean formar parte activa de una sociedad en tantas otras ocasiones descorazonadora.

Para ello es imprescindible la provocación en el ejercicio de la reflexión personal. Los procesos emancipatorios, que diría Giroux (2001), son procesos conscientes y necesarios en los contextos en los que convivimos. Si algo caracteriza a nuestra sociedad es su carácter dinámico en una evolución a veces desmedida y absolutamente imparable. Enfatizar el desarrollo de la crítica y el distanciamiento de aquello que nos viene dado, son objetivos completamente justificados y claramente beneficiosos desde la perspectiva transformadora de la educación y, por supuesto, también desde la animación e implicación sociocultural.

El principio de independencia necesita para ser "de facto" invocar a la práctica del razonamiento sin que por ello dejemos de ser naturalezas “sintientes", tal y como afirmaría Edgar Morín (2001). Estamos de acuerdo con que el afecto no está reñido con la razón, pero en muchas ocasiones es preferible calibrar ambos. Convendría, entonces, tomar conciencia de las necesidades que existen (o que sentimos) en el contexto del cual formamos parte, pero también repensar hasta qué punto somos influenciados por él.

La participación socio-comunitaria no escapa a los instrumentos de dominación social y cultural imperantes, sin embargo la crítica y la conciencia nos ayudan en el quehacer del pensamiento y el discernimiento. Esta tarea desembocaría en una de las funciones más comprometidas: el cambio estructural e incluso la transformación social. Pero antes hemos de comenzar por facilitar de forma individual los procesos de cuestionamiento y reflexión personal que anteceden al principio de independencia. No obs- 
tante, concretando el discurso al tema que nos ocupa, no es posible anclarnos en planteamientos únicamente individualistas. La Carta Internacional para la Educación del Ocio, antes citada, afirma en este sentido que «los requisitos y condiciones para el ocio no pueden asegurarse de manera individual»..... "La educación del ocio es un proceso continuo de aprendizaje que incorpora el desarrollo de actitudes, valores, conocimiento, habilidades y recursos de ocio”. Y continúa definiendo los objetivos de la educación del ocio en la comunidad, entre los que destaca la capacitación, accesibilidad, aprendizaje de por vida, participación social, disminución de impedimentos, inclusión, responsabilidad cívica y moral y preservación.

Lo expresado hasta aquí confluye en el valor del método que defendemos para las personas de más edad: el método interactivo. Dentro de la filosofía que persigue el desarrollo comunitario, entendiendo como tal un trabajo destinado a compensar necesidades sociopersonales y en el que participan los propios afectados, parece conveniente no tomar a la ligera el papel que desempeñan cada uno de los participantes. En realidad, es un proceso gradual y complejo dirigido hacia un creciente autodescubrimiento y compromiso.

La implicación personal en un contexto social pasa a ser referente básico de actuación a través del método interactivo, conteniendo las siguientes etapas:

- Una clarificación acerca de las ideas, capacidades y deseos.

- Un encuentro consigo mismo: qué elementos y acontecimientos han repercutido en mi vida para considerarme tal cual soy.

- Un contraste de posicionamiento con los otros: cuáles son mis decisiones y qué actitudes personales determinan una postura concreta frente a las mismas; cuáles son mis valores, mis convicciones y mis actitudes prioritarias vistas por las demás personas del grupo.

- Un cuestionamiento particular: pasar a un proceso de introspección personal. Reflexionar acerca de lo que he descubierto sobre mí y si se corresponde con lo que deseo conseguir en el grupo: qué quiero y puedo ser y hacer.

- El establecimiento de conclusiones: cuál es mi compromiso social, hacia dónde me encamino.

Bajo un paradigma crítico, pasar de la reflexión a la acción conlleva un auto-cuestionamiento pero también un acercamiento a la realidad, una clarificación de los propios valores, una conciencia clara de aquello que puedo y quiero conseguir y una apuesta por modificar, en la medida en que las posibilidades y circunstancias lo permitan, mi propio entorno. Sin embargo, como hemos venido exponiendo, hemos de ser realistas y reconocer la evidencia: limitados y condicionados por múltiples circunstancias (so- 
cio-históricas, económicas, políticas, personales...), la cuestión estriba en la dificultad de toda persona a la hora de disponer y organizar el tiempo según sus propias determinaciones. Partiendo de ello, habrá que seguir encarando los elementos que posibiliten la efectiva participación e implicación, incluyendo los que tienen que ver con el disfrute del tiempo libre, actividades de ocio y voluntariado como tareas de gran trascendencia y (re)valorización socio-comunitaria, al menos dentro de los contextos y posibilidades que así lo permitan. Porque, tal como afirma el profesor Csikszentmihalyi (2004: 374), por dotada que esté una persona no tiene posibilidad alguna de conseguir nada creativo a menos que el ámbito ofrezca las circunstancias adecuadas: formación, expectativas, recursos, reconocimiento, esperanzas, oportunidades y recompensas. Siguiendo este razonamiento, el pensamiento creativo y el desarrollo del ocio se pueden incrementar de forma sustancial, siempre que la misma sociedad genere mayores posibilidades reales que generen y favorezcan otros estilos de vida. Fundamentar su conveniencia, demandarlos como ejercicio real y convertirlos en instrumento y objetivo socio-educativo, también es nuestra responsabilidad como educadores. 


\section{Bibliografía}

Águila, C. (2007), Sobre el ocio y la posmodernidad, Wanceulen, Sevilla.

Bazo, M $\mathrm{M}^{\mathrm{a}} \mathrm{T}$. (coord.) (1999), Envejecimiento y sociedad: una perspectiva internacional, Editorial Panamericana, Madrid.

Bedmar, M. y Montero, I. (coords.) (2003), La educación intergeneracional: un nuevo ámbito educativo, Dykinson, Madrid.

Belsky, J. K. (1996), Psicología del envejecimiento. Teoría, investigaciones e intervenciones, Editorial Masson, S.A., Barcelona.

Bermejo, L. y otros (2010), Envejecimiento activo y actividades socioeducativas con personas mayores. Guía de buenas prácticas, Editorial Médica Panamericana, Madrid.

Castro, A. de (1990), La Tercera Edad: tiempo de ocio y cultura, Narcea, Madrid.

Csikszentmihalyi, M.; Cuenca, M. y otros (2001), Ocio y desarrollo. Potencialidades del ocio para el desarrollo humano, Universidad de Deusto, Bilbao.

Csikszentmihalyi, M. (2004), Creatividad. El fluir y la psicología del descubrimiento y la invención, Paidós, Barcelona.

Cuenca, M. (1995), Temas de Pedagogía del Ocio, Universidad de Deusto, Bilbao.

Idem, (1999), Ocio y formación: hacia la equiparación de oportunidades mediante la educación de ocio, Universidad de Deusto, Bilbao.

Idem, (2004), Pedagogía del ocio: Modelos y propuestas, Universidad de Deusto, Bilbao.

Dávila, C. y Díaz, J.F. (2009), "Voluntariado y tercera edad”, Anales de psicología, vol. 25, diciembre, $\mathrm{n}^{\circ}$ 2, pp. 375-389.

Del Barrio, E. (2007), “Uso del tiempo entre las personas mayores”, Perfiles y tendencias. Boletín sobre el envejecimiento, marzo, $\mathrm{n}^{\circ}$ 27, pp. 1-40.

Dumazedier, J. (1968), Hacia una civilización del ocio, Estela, Barcelona.

Edginton, C. (2007), "The world leisure organization: promoting social, cultural and economic transformation", en Licere, Belo Horizonte, Volumen 10, $\mathrm{n}^{\circ}$ 2, ago. 2007. Disponible en: http://www.eeffto.ufmg.br/licere/ pdf/licereV10N02_a2.pdf 
Elizalde, R. (2010), “Resignificación del ocio: aportes para un aprendizaje transformacional”, Polis, Revista de la Universidad Bolivariana, Volumen 9, $\mathrm{N}^{\circ}$ 25, 2010, pp. 437-460. Disponible en: http://redalyc.uaemex.mx/ redalyc/src/inicio/ArtPdfRed.jsp?iCve $=30512376026$

Erlinghagen, M. y Hank, K. (2005), “Participación de las personas mayores europeas en el trabajo de voluntariado”, Perfiles y tendencias. Boletín sobre el envejecimiento, agosto, $\mathrm{n}^{0} 17$, pp. 1-24.

Fantova, F. (2002), “Ocio, personas mayores y voluntariado”, Adoz. Boletín del Centro de Documentación en Ocio (Universidad de Deusto/Instituto de Estudios de Ocio), $n^{\circ}$. 24, pp. 48-52.

Fernández, G. (2001), “La ciudadanía en el marco de las políticas educativas”, Revista Iberoamericana de Educación, núm. 26, Madrid, OEI. Disponible en: http://www.rieoei.org/rie26a08.htm

García Mínguez, J. (2004), La educación en personas mayores. Ensayo de nuevos caminos, Narcea, Madrid.

Gil Calvo, E. (1995), “Elogio del ocio”, Temas para el Debate, n 9-10, pp. 26-29.

Giró, J. (2009) (coord.), Envejecimiento, tiempo libre y gestión del ocio, Universidad de La Rioja, Logroño.

Giroux, H. A. (2001), Cultura, política y práctica educativa, Graó, Barcelona.

Gomes, C y Elizalde, R. (2009), “Trabajo, tiempo libre y ocio en la contemporaneidad: Contradicciones y desafíos”, Polis. Revista de la Universidad Bolivariana, Volumen 8, $\mathrm{N}^{\circ}$ 22, 2009, p. 249-266. Disponible en: http://www.scielo.cl/pdf/polis/v8n22/art15.pdf

Hernández Rodríguez, G. (2009), “Jubilación y alternativas para un ocio creativo”, en J. Giró, (coord.), Envejecimiento, tiempo libre y gestión del ocio, Universidad de La Rioja, Logroño.

Leif, J. (1992), Tiempo libre y tiempo para uno mismo. Un reto educativo y cultural, Narcea, Madrid.

Limón, Ma R. (2004), “Educación para el ocio y tiempo libre en las personas mayores”, en G. Pérez, (coord.), ¿Cómo intervenir en personas mayores? Dykinson, Madrid, pp. 139-174.

Maizel, S. (1987), “Good deeds in old age: volunteering by the new leisure class”, Lexington Books, Toronto.

Martín, M. (2006), “Conocer, manejar, valorar, participar: Los fines de 
una educación para la ciudadanía”, Revista Iberoamericana de Educación, núm. 42, Madrid, OEI. Disponible en: http://www.rieoei.org/rie42a04.htm

Melendez, N. (1991), ¿Contribuye la recreación a la satisfacción del envejeciente jubilado?, Universidad de Puerto Rico, Puerto Rico.

Montero I. (2003), “El caletre y la experiencia”, en J. García Mínguez, (coord.): El refranero, ¿espejo y reflejo de las personas mayores? Dykinson, Madrid, pp. 155-170.

Morín, E. (2001), Los siete saberes necesarios para la educación del futuro, Paidós, Barcelona.

Navajo, P. (2004), La promoción del voluntariado de las personas mayores. Disponible en www.iniciativasocial.net/tercera.htm

Núñez, V. (2003), “El vínculo educativo”, en Hebe Tizio (coord.), Reinventar el vínculo educativo: aportación 